\title{
Ethnobotanicals used for the Treatment of Skin Diseases with Special Emphasis on Carbuncle Disease from Purulia District of West Bengal in India
}

\author{
Ghanashyam Mahato', Bangamoti Hansda ${ }^{2}$, Nilanjana Banerjee ${ }^{3, *}$
}

Ghanashyam Mahato', Bangamoti Hansda ${ }^{2}$, Nilanjana Banerjee ${ }^{3, *}$

${ }^{1}$ Assistant Professor, Department of Botany, A.M. College, Jhalda, Purulia, West Bengal-723202, INDIA.

${ }^{2}$ Research Scholar, Department of Botany and Forestry, Vidyasagar University,

Midnapore, West Bengal-721102, INDIA.

${ }^{3}$ Assistant Professor, Department of Botany, Vidyasagar University, Paschim Medinipur, West Bengal-721102, INDIA.

\section{Correspondence}

\section{Nilanjana Banerjee}

Assistant Professor, Department of Botany, Vidyasagar University, Paschim Medinipur, West Bengal-721102, INDIA.

Phone no: 919836960317;

E-mail: nilanjanab1@yahoo.com

History

- Submission Date: 13-10-2018;

- Review completed: 06-04-2019;

- Accepted Date: 21-04-2019.

DOI : 10.5530/pj.2019.11.118

Article Available online

http://www.phcogj.com/v11/i4

\section{Copyright}

(C) 2019 Phcogj.Com. This is an openaccess article distributed under the terms of the Creative Commons Attribution 4.0 International license.

\begin{abstract}
Background: From time immemorial ethnic people of Purulia district of West Bengal are well acquainted with different plant resources for the treatment of various skin diseases including 'carbuncle' and others skin diseases. Carbuncle, caused by the methicillin resistant Staphylococcus aureus is of major concern in this part of India, mostly dominated by tribes like Majhi, Munda, Santal, Birhor, Ho and Rajwar etc. Hot climate, overcrowded households, improper sanitation, very poor economic background and frequent burn accidents are of major issues for spreading of these bacterial infections. Objective: Present authors are trying to summarize these ethno-medicinal knowledge of the local, conservative traditional healers by using structured questionnaires given to them and are trying to analyze these information from scientific perspective. Materials and Methods: Plant samples were collected from March 2014 to May 2016, mostly during their flowering stage and a total of 62 people, both male $(84 \%)$ and female (16\%) were interviewed. Informant consensus factor, fidelity level and use value were calculated. Results: Fifty-nine herbal plants belongs to 35 families were recorded for the cure of various skin diseases, among which 10 plants are used individually during medication whereas 9 polyherbal formulations were used in various combinations during treatment. Five species of the families Moraceae and Asteraceae was found to be the most common medicinal plants, among which $44.89 \%$ are herbaceous in habit. Leaves $(55.55 \%)$ are the most common plant part for their use. Conclusion: Therefore, the present paper has been written to document this rapidly vanishing huge knowledge of folklore which should be digitally conserved for futuristic approach on medicinal plants in India.

Key words: Ethnobotany, Carbuncle, Purulia, Use value, Informant consensus factor, Fidelity level.
\end{abstract}

\section{INTRODUCTION}

In ancient India plants were widely used for the treatment of skin disorders. In many countries till today, medicinal plants contribute significantly in the primary health care system of the rural population. ${ }^{1-4}$ One of the most important skin ailments, mostly found in the tribal population of Purulia is carbuncle disease which is caused by the bacteria Staphylococcus aureus. However, the presence of carbuncle is actually the sign of active system to resist other skin infections. ${ }^{5}$ The infection is contagious and may spread to other areas of the body. Each year, around 500,000 patients in hospitals of the United States are attacked by Staphylococcal infection, chiefly by $S$. aureus. ${ }^{6}$ Since discovery $S$. aureus is experimentally resistant to a lot of antibiotics for example penicillin group (methicillin, oxacillin and cloxacillin), ${ }^{7,8}$ amino glycosides, macrolides, tetracycline, chloramphenicol and lincosamides. Vancomycinresistant $S$. aureus (VRSA) is a strain of $S$. aureus that usually resistant to the glycopeptides. The first case of vancomycin-intermediate S. aureus (VISA) was reported in Japan in $1996 .{ }^{9}$

Since last two decades development of drug resistance as well as the appearance of undesirable side effects of certain antibiotics lead us to search for new chemical structures having antimicrobial property from plant extracts. ${ }^{10}$ Although the people of Purulia traditionally used various herbal plants for preparing drugs and medicines to treat carbuncle and some skin diseases no such detail documentation has been done earlier. Hence, the primary objective of the present study is to investigate the folkloric wisdom practiced by the aboriginals residing in the Purulia district, one of the poorest district in India, against skin diseases with special emphasis on carbuncle.

\section{MATERIALS AND METHODS}

\section{Study area}

The study area is located in the western most part of West Bengal, India (Figure 1). This area is situated between $22^{\circ} 6^{\prime}$ to $23^{\circ} 5^{\prime} \mathrm{N}$ latitude and $85^{\circ} 7^{\prime}$ to $86^{\circ} 6^{\prime}$ E longitude and has an area of $6259 \mathrm{~km}^{2}$. Purulia district covers subtropical ecology with deciduous forest mainly with luxurious population of Sal, Palash, Piyal, Mohul etc. having high diurnal temperature during summer (upto $52^{\circ} \mathrm{C}$ ) and with average rainfall $1300 \mathrm{~mm}$. It is situated under Chotonagpur plateau having undulated topography and lateritic soil.

Cite this article: Mahato G, Hansda B, Banerjee N. Ethnobotanicals used for the Treatment of Skin Diseases with Special Emphasis on Carbuncle Disease from Purulia District of West Bengal in India. Pharmacog J. 2019;11(4):745-53. 


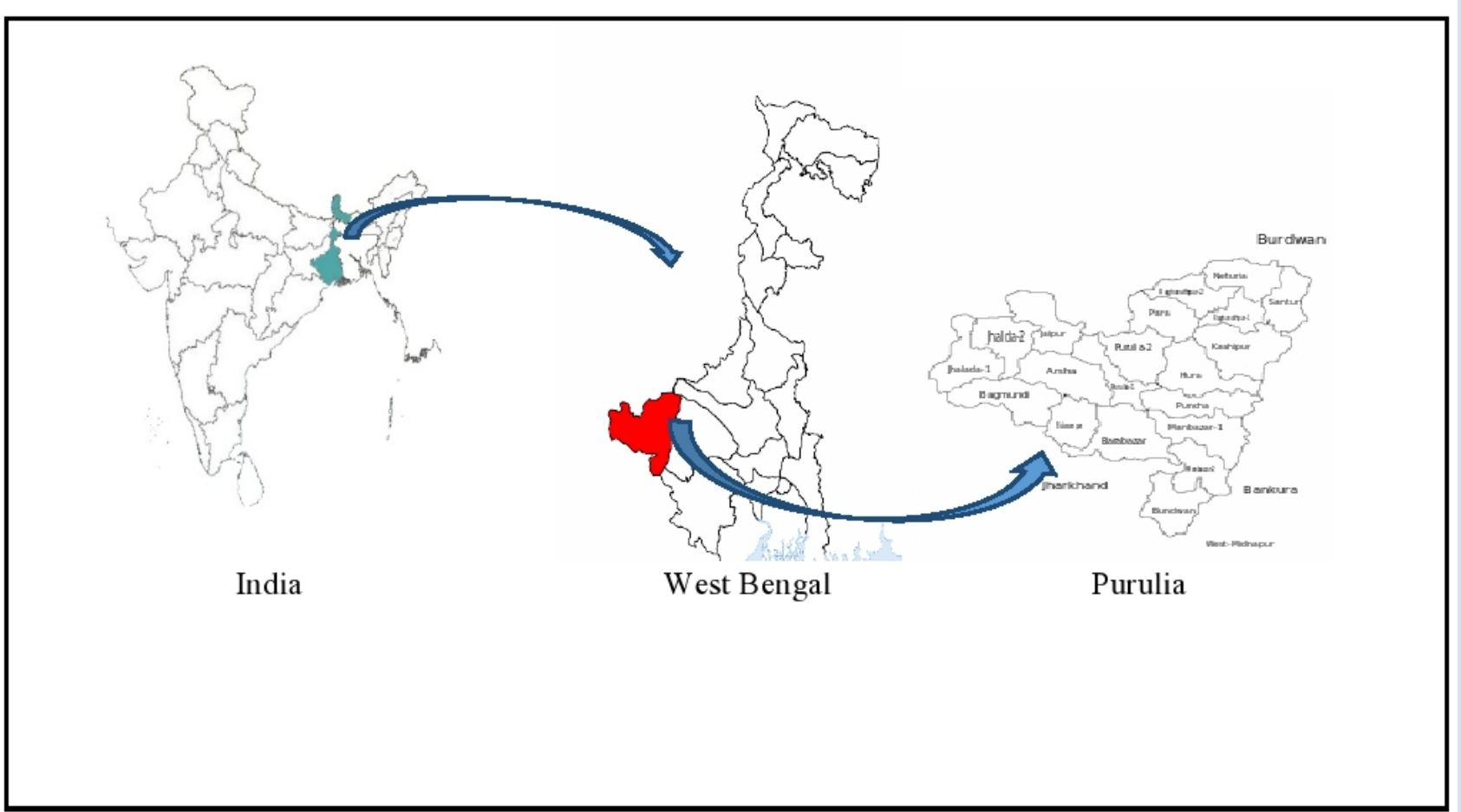

Figure 1: Map of study area. (not in scale).

\section{Ethnobiological data collection and analyses}

The survey was carried out from 20 field session based on ethnobiological explorations from 2014 to 2016. Main emphasis was given to the Maoist hot parts of the district. As GM, first author, knows some of the native languages of the tribes, semi structured questionnaire were placed to the informants following a standard ethno botanical method in local vernacular or in Bengali. ${ }^{11,12}$ Herbarium specimens of these medicinal plants mostly in their flowering stage were collected for future references. The medicinal plant specimens were identified with the help of authentic herbarium specimens of Botanical survey of India, books, floras and revisions ${ }^{13-16}$ and preserved, recorded and documented in the herbarium of Department of Botany, A. M. College, Jhalda.

Descriptive statistics was applied to compute the number and percentage of species, genera and families of ethnomedicinal plants, proportions of plant parts harvested, plant percentage from various sources, plant distribution among different families, life forms, nature of habitat and plant percentage in curing various ailments. Key informants are mostly more than fifty years old and are respectable person of that locality (Figure 3). The collected data was analyzed with three quantitative tools viz. the informant consensus factor (Fic), fidelity level (FL) and use value (UV). To test the level of homogeneity of information provided by different informants, Informants' Consensus Factor $\left(F_{\text {ic }}\right)$ was calculated $.{ }^{17} \mathrm{Fic}=$ Nur-Nt/ $($ Nur-1) (where,Nur $=$ number of use reports in each disease category; $\mathrm{Nt}=$ number of times species used). The value ranges from 0 to 1 . High Fic value (close to1) means there exist well-defined selection for the species on account of a specific disease category whereas low Fic values (close to 0 ) indicates there is lack of consensus amongst the informants related to the medicinal uses of the species. Fidelity level (FL) inde ${ }^{18}$ was used to determine the relative healing potential of each reported medicinal plant used against various ailments. Fidelity level $(\mathrm{FL} \%)=(\mathrm{Np} / \mathrm{N} x 100)($ where $\mathrm{Np}=$ the number of informants who independently indicated the use of a species for treating a particular disease and $\mathrm{N}=$ total number of informants who reported the plant for any given disease). The use value (UV $)^{19}$ was also calculated using the formula: $U V=(\Sigma U / n)$, where $U V$ is the use value of species, ' $U$ ' is the total number of use reports per species and ' $n$ ' represents the total number of informants interviewed for a given plant. Values ranges from near1 to 0 . High UV means there are many use reports for a specific plant and that plant is marked important for treatment.

\section{RESULTS}

\section{Ethnomedicinal plant diversity and uses reported by the informants}

Medicinal plants was enlisted with scientific name and author citation, followed by local name, family, habit, plant part (s) used and ailment (s) against each disease (Table 1). A total number of 10 monoherbal formulations (MF) were recorded which were used singly for medication during treatment and 9 polyherbal formulations (PF) were used in combination by different ethnic groups of this area (Table 2). The highest number of medicinal plants were recorded in four families viz. Moraceae (5 species), Asteraceae (5 species), Amaranthaceae (4 species), Euphorbiaceae (3 species), Fabaceae (3 species) and Malvaceae (3 species), followed by families Amaryllidaceae, Asclepiadaceae, Solanaceae and Myrtaceae. Each of these families comprises two species.

The distribution of plant habit types, plant part (s) used and method of preparations are illustrated in Figures 2-4 respectively. Out of 59 species $44.89 \%$ herbaceous plants are mostly used for medication followed by $36.73 \%$ trees, $14.28 \%$ shrubs and $2.04 \%$ climbers (Figure 2). Leaves (55.55\%) were found to be the most favored plant parts followed by roots (11.11\%), whole plant (5.55\%) and barks (5.55\%) (Figure 3). Quantitative ethno botanical analyses revealed high UV for Curculigo orchioides Gaertn. (0.81), Hibiscus rosa-sinsnsis L. (0.79), Urginea indica (Roxb). Kunth (0.77), Glossocardia bidens (Retz) Veldkamp (0.88), Smilax zeylanica L. (0.82), Elephantopus scaber L. (0.72) etc. Highest Fic value was represented by chicken pox (1), carbuncle (0.91) and ulcer (0.87) whereas lowest Fic value was exhibited mouth ulcer (0) and nail 


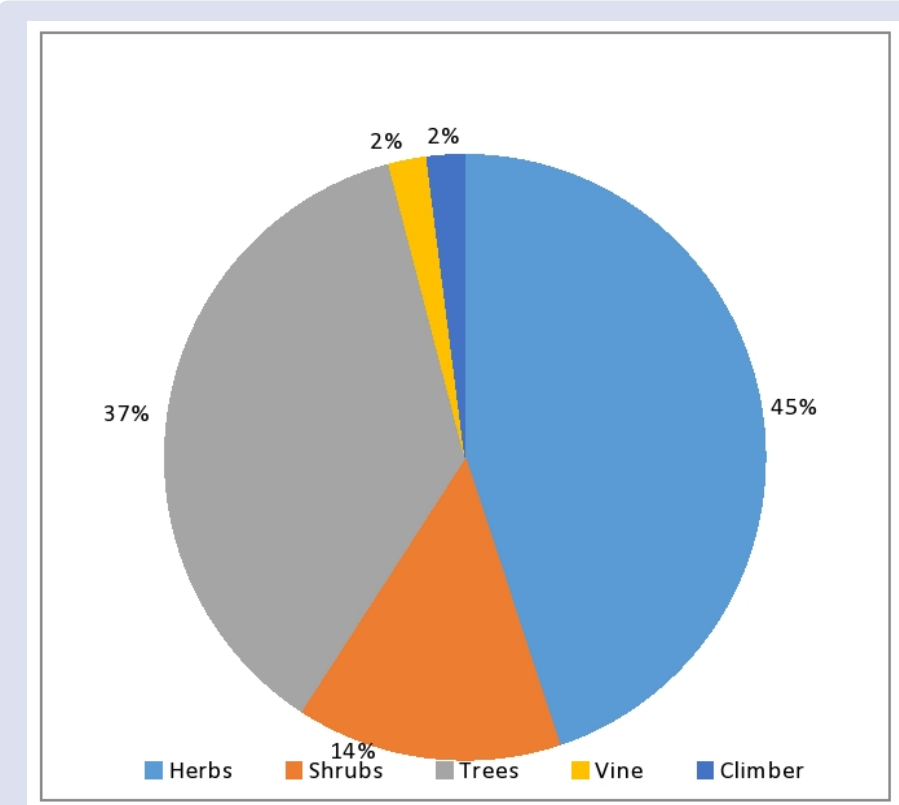

Figure 2: Percentage of habit types of the total studied plant specimens used for the treatment of carbuncle.

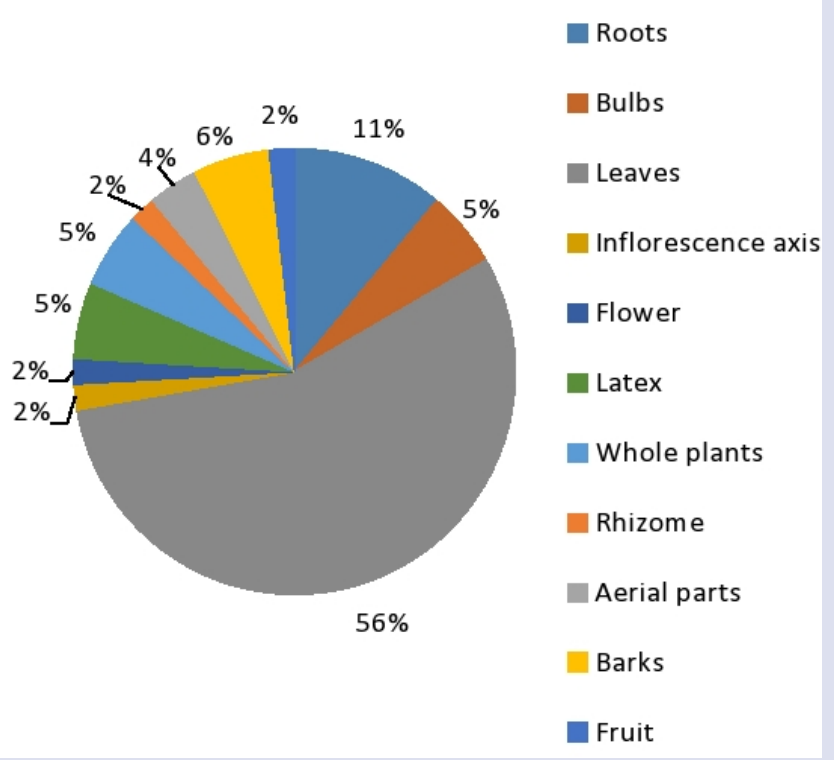

Figure 3: Percentage of plant parts used for medication.

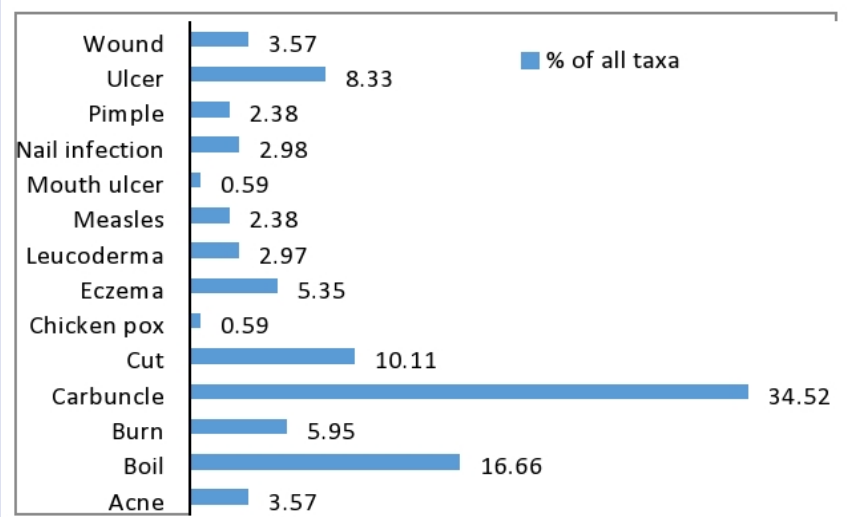

Figure 4: Percentage of species used for the treatment of a particular disease. 
Table 1: Ethnobotanicals used for carbuncle and some other skin diseases treatment.

\begin{tabular}{|c|c|c|c|c|c|c|c|}
\hline SI. No. & Latin name & Local Name & Family & Plant parts used & $\begin{array}{l}\text { Ailments other than } \\
\text { Carbuncle }\end{array}$ & UV value & Habit \\
\hline 1 & Achyranthes aspera L. & Chitchiti & Amaranthaceae & Roots & Boi and Ulc & 0.4 & Herb \\
\hline 2 & Achyranthes bidentata Blume & Chitni & Amaranthaceae & Roots & Mea & 0.24 & Herb \\
\hline 3 & Aerva sanguinolenta(L.) Blume & Chaldhuya & Amaranthaceae & Aerial parts & Mea and Cut & 0.42 & Herb \\
\hline 4 & Ageratum conyzoides $L$. & Bhabri & Asteraceae & Leaves & Cut,Boi & 0.24 & Herb \\
\hline 5 & Allium sativum $\mathrm{L}$. & Piyaj & Amaryllidaceae & Bulbs & Boi and Ulc & 0.33 & Herb \\
\hline 6 & Aloe vera $\mathrm{L}$. & Ghritakumari & Xanthorrhoeaceae & Leaves & $\begin{array}{l}\text { Ulc, Cut,Acne and nail } \\
\text { infection }\end{array}$ & 0.54 & Herb \\
\hline 7 & Amaranthus spinosus L. & kata nate & Amaranthaceae & Leaves & Boi,Acne & 0.22 & Herb \\
\hline 8 & $\begin{array}{l}\text { Andrographis paniculta (Burm.f.) Wal. } \\
\text { ex. Nes. }\end{array}$ & Kalmegh & Acanthaceae & Leaves & Ulc & 0.43 & Herb \\
\hline 9 & Annona squamosa L. & Atapata & Annonaceae & Leaves & Boi and Pim & 0.29 & Tree \\
\hline 10 & Argemone mexicana Linn & Siyalkata & Papaveraceae & Roots & Cut & 0.21 & Herb \\
\hline 11 & Artocarpus heterophyllus Lam. & Kathal & Moraceae & $\begin{array}{l}\text { Inflorescence } \\
\text { axis(IA) }\end{array}$ & Boi and Leo & 0.39 & Tree \\
\hline 12 & Averrhoa carambola L. & Kamranga & Oxalidaceae & Roots & Ulc & 0.19 & Tree \\
\hline 13 & Azadirachta indica A. Juss. & $\mathrm{Nim}$ & Meliaceae & Leaves & $\begin{array}{l}\text { Mea, Chick pox, Ulc } \\
\text { and Boi }\end{array}$ & 0.75 & Tree \\
\hline 14 & Barleria lupulinaLindl. & Bialyakaran & Acanthaceae & Leaves & Bur & 0.26 & Shrub \\
\hline 15 & Bauhinia purpurea $\mathrm{L}$. & kanchan & Leguminosae & Leaves & Boi and Bur & 0.22 & Shrub \\
\hline 16 & Boerhavia diffusa L. & Kathasak & Nyctaginaceae & Leaves & Cut & 0.39 & Herb \\
\hline 17 & Bombax ceiba L. & Bakul & Bombacaceae & Leaves & Mouulc & 0.21 & Tree \\
\hline 18 & Bryophyllum pinnatum (Lam)Oken & Patharkuchi & Crassulaceae & Leaves & Boi & 0.26 & Herb \\
\hline 19 & Calotropis gigantea (Linn.) R. Br.ex.Ait. & Akuni & Asclepidaceae & Leaves and Latex & Bur & 0.37 & Shrub \\
\hline 20 & Calotropis Procera (Aiton) R. Br. & Akanda & Asclepidaceae & Leaves & Bur & 0.26 & Shrub \\
\hline 21 & Cannabis sativa $\mathrm{L}$. & Ganja & Cannabaceae & Leaves & Cut & 0.12 & Shrub \\
\hline 22 & Cotula anthemoides L. & Tar dingla & Asteraceae & Whole plant & $\begin{array}{l}\text { Ulc,Boi,Acne,Leo and } \\
\text { Pim }\end{array}$ & 0.88 & Herb \\
\hline 23 & Crinum asiaticum Linn. & Baniyaj & Amaryllidaceae & Bulbs & Boi & 0.48 & Herb \\
\hline 24 & Curculigo orchioides Gaertn. & Talmuli & Hypoxidaceae & Roots & Boi,Bur,Leo and cut & 0.81 & Herb \\
\hline 25 & Curcuma longa L. & Halud & Zingiberaceae & Rhizomes & Ulc, Cut and Acne & 0.52 & Herb \\
\hline 26 & Cuscuta reflexa Roxb. & Sarnalata & Convolvulaceae & Whole plant & Boi & 0.47 & Climber \\
\hline 27 & Cyperus rotundus $\mathrm{L}$. & Mutha & Cyperaceae & Whole plant & Ulc & 0.47 & Herb \\
\hline 28 & Datura metel L. & Dhutra & Solanaceae & Leaves & Nail inf & 0.15 & Shrub \\
\hline 29 & Datura stramonium L., & Dhutrajhuri & Solanaceae & Leaves & Nail inf & 0.09 & Shrub \\
\hline 30 & Elephantopus scaber L. & Mayurjhuti & Asteraceae & Roots & Boi,Bur and Ulc & 0.42 & Herb \\
\hline 31 & Euphorbia hirta L., & Lalpata & Euphorbiaceae & Aerial parts & Mea and Cut & 0.42 & Herb \\
\hline 32 & Euphorbia milii var. longifolia D. Moul. & Latjhakha & Euphorbiaceae & Leaves & Bur & 0.16 & Herb \\
\hline 33 & Ficus benghalensis L. & Asasta & Moraceae & Leaves and Bark & Pim & 0.1 & Tree \\
\hline 34 & Ficus hispida L. f. & Dumur & Moraceae & Leaves and Latex & Boi and Bur & 0.27 & Tree \\
\hline 35 & Ficus racemosa $\mathrm{L}$. & Pagadumur & Moraceae & Leaves and Latex & Boi and Bur & 0.39 & Tree \\
\hline 36 & Ficus religiosa $\mathrm{L}$. & Jar & Moraceae & Leaves & Nail inf & 0.03 & Tree \\
\hline 37 & Gloriosa superba Linn. & Barphuli & Colchicaceae & Leaves & Leo & 0.22 & Herb \\
\hline 38 & Glossocardia bidens (Retz) Veldkamp & Pisainandi & Asteraceae & Whole plant & $\begin{array}{l}\text { Ulc,Boi,Acne,Leo and } \\
\text { Pim }\end{array}$ & 0.88 & Herb \\
\hline 39 & Gmelina arborea Roxb. ex Sm. & Gamer & Lamiaceae & Barks & Wou and cut & 0.24 & Tree \\
\hline 40 & Hemidesmus indicus (L.) R.Br. & Anantamuli & Apocynaceae & Aerial parts & Ecz and Ulc & 0.47 & Herb \\
\hline 41 & Hibiscus rosa-sinensis $\mathrm{L}$. & Jaba & Malvaceae & Leaves & Boi and Acne & 0.79 & Shrub \\
\hline 42 & Lawsonia inermis L. & Natur & Lythraceae & Leaves & Cut & 0.21 & Shrub \\
\hline 43 & $\begin{array}{l}\text { Lippia alba (Mill.)N.E.Br.ex Britton and } \\
\text { P.Wilson }\end{array}$ & laltia & Verbenaceae & Aerial parts & $\mathrm{Ecz}$ & 0.47 & Herb \\
\hline 44 & Madhuca longifolia (Konig) J.F.Macbr. & Mahua & Sapotaceae & Flower & Boi, Ulc and Bur & 0.58 & Tree \\
\hline 45 & Magnifera indica L. & Aam & Anacardiaceae & Leaves & Ecz and wou & 0.4 & Tree \\
\hline 46 & Piper betle L. & Pan & Piperaceae & Leaves & Boi & 0.5 & Vine \\
\hline 47 & $\begin{array}{l}\text { Polygala crotalarioides Buch.-Ham. } \\
\text { ex Dc. }\end{array}$ & Nilkantha & Polygalaceae & Root & Boi & 0.45 & Herb \\
\hline 48 & Psidium guajava L. & Peyara & Myrtaceae & Leaves & Ecz and Nail inf & 0.19 & Tree \\
\hline 49 & Pterocarpus santalinus L.f. & Set chandan & Fabaceae & Barks & Boi,Ecz and Wou & 0.64 & Tree \\
\hline 50 & Ricinus communis $\mathrm{L}$. & Jara & Euphorbiaceae & Leaves & Boi and cut & 0.4 & Tree \\
\hline 51 & Shorea robusta Gaertn. & Sal & Dipterocarpaceae & Leaves & Boi,Ecz and Wou & 0.42 & Tree \\
\hline
\end{tabular}




\begin{tabular}{|c|c|c|c|c|c|c|c|}
\hline SI. No. & Latin name & Local Name & Family & Plant parts used & $\begin{array}{l}\text { Ailments other than } \\
\text { Carbuncle }\end{array}$ & UV value & Habit \\
\hline 52 & Sidacordata (Burm. f.) Borss. Waalk. & Laltoa & Malvaceae & Leaves & $\mathrm{Ecz}$ & 0.32 & Herb \\
\hline 53 & Sida cordifolia L. & Chalpata & Malvaceae & Leaves & Boi and cut & 0.48 & Herb \\
\hline 54 & Smilax zeylanica L. & Ramdatun & Smilacaceae & Roots & Boi,Ecz and Wou & 0.42 & Shrub \\
\hline 55 & Syzygium cumini (L) Skeels & Jam & Myrtaceae & Leaves & Wou and cut & 0.63 & Tree \\
\hline 56 & Tagetes patula $\mathrm{L}$. & Gandha & Asteraceae & Leaves & Cut and Ecz & 0.6 & Herb \\
\hline 57 & Tamarindus indica $\mathrm{L}$. & Tetul & Leguminosae & Leaves and Fruits & Boi and Ulc & 0.73 & Tree \\
\hline 58 & Urginea indica (Roxb.)Kunth & Banpiyaj & Liliaceae & Bulbs & Boi,Leo, Cut and Ulc & 0.77 & Herb \\
\hline 59 & Vitex negundo L. & Buyan & Verbenaceae & Leaves & Cut and Boi & 0.61 & Shrub \\
\hline
\end{tabular}

Wou = wound Boi = Boil; Cut = Cut Ulc = Ulcer Leo = Leukoderma Ecz = Eczema Nail inf = Nail infection; Pim = Pimple; Mea = measles; Moulc = Mouth ulcer; Chick pox = Chicken pox; Bur = Burn; Acn = Acne;Car = Carbuncle.

\section{Table 2: Details of formulations for medication.}

\begin{tabular}{|c|c|c|c|c|c|}
\hline Formulations & $\begin{array}{l}\text { Plant species used } \\
\text { singly / combinations }\end{array}$ & Parts used & Additives & Method of preparation & $\begin{array}{c}\text { Mode of } \\
\text { administration }\end{array}$ \\
\hline MF1 & A vera & $\mathrm{L}$ & Water & Paste & $\begin{array}{l}\text { Apply on } \\
\text { Carbuncle }\end{array}$ \\
\hline MF2 & A indica & $\mathrm{L}$ & Water & Form small pea sized pills & $\begin{array}{l}\text { Once a day } \\
\text { before breakfast }\end{array}$ \\
\hline MF3 & C gigantea & $\mathrm{L}+\mathrm{LA}$ & NR & Burn to form ash & $\begin{array}{l}\text { Apply on mouth } \\
\text { of Carbuncle }\end{array}$ \\
\hline MF4 & D metel & $\mathrm{L}$ & NR & Burn to form ash & $\begin{array}{l}\text { Apply on mouth } \\
\text { of Carbuncle }\end{array}$ \\
\hline MF5 & D stramonium & $\mathrm{L}$ & Milk; Rhizome of kanchahalud/turmaric & Paste & $\begin{array}{l}\text { Apply on } \\
\text { Carbuncle }\end{array}$ \\
\hline MF6 & V negundo & $\mathrm{L}$ & Jira/Cumin seeds & Paste & $\begin{array}{l}\text { Apply at starting } \\
\text { time }\end{array}$ \\
\hline MF7 & $C$ anthemoides & WH & Honey & Form small sized pills & $\begin{array}{l}\text { Three times in a } \\
\text { day before meal }\end{array}$ \\
\hline MF8 & C viviparum & BU & Milk from sheep & paste & $\begin{array}{l}\text { Apply on mouth } \\
\text { for seven days }\end{array}$ \\
\hline MF9 & Corchioides & RH & Water & Form small pea sized pills & $\begin{array}{c}\text { Two times } \\
\text { before to take } \\
\text { meal }\end{array}$ \\
\hline MF 10 & G bidens & WH & Water & Paste & $\begin{array}{l}\text { Applied on the } \\
\text { affected parts } \\
\text { twice a day for } \\
\text { five days }\end{array}$ \\
\hline PF1 & $\begin{array}{c}\text { A aspera }+ \\
\text { A sativum }+ \\
\text { A paniculta+ } \\
\text { A squamosa+ } \\
\text { A heterophyllus }+ \\
\text { A indica+ } \\
\text { C viviparum }+ \text { G bidens }+ \\
\text { A sanguinolenta }\end{array}$ & $\begin{array}{c}\mathrm{R}+\mathrm{BU}+\mathrm{L}+\mathrm{L}+\mathrm{IA} \\
+\mathrm{L}+\mathrm{BU}+\mathrm{A}+\mathrm{A}\end{array}$ & Talmichri made from palm tree; Honey & $\begin{array}{l}\text { Grinding all the items, small pea- } \\
\text { sized pills are made from a paste }\end{array}$ & $\begin{array}{l}\text { Once in a day } \\
\text { for five days }\end{array}$ \\
\hline PF2 & $\begin{array}{c}\text { A carambola+ } \\
\text { C Procera+ } \\
\text { C longa }+ \\
\text { E scaber }+ \\
R \text { communis }+ \\
\text { A vera }+ \\
C \text { anthemoides }+ \\
A \text { indica }+L \text { alba }\end{array}$ & $\begin{array}{c}\mathrm{R}+\mathrm{L}+\mathrm{Rh}+\mathrm{R}+\mathrm{L} \\
+\mathrm{L}+\mathrm{WH}+\mathrm{L}+\mathrm{L}\end{array}$ & Kalajira; Mirchi & $\begin{array}{l}\text { After grinding all the ingredients } \\
\text { pills are prepared from a paste }\end{array}$ & Thrice a day \\
\hline PF3 & $\begin{array}{c}\text { F recemosa+ } \\
\text { H rosa-sinsnsis } \\
+P \text { guajava } \\
+S \text { cordata } \\
+S \text { cumini }+ \\
\text { C anthemoides }+S \\
\text { zeylanica }+A \text { conyzoides }\end{array}$ & $\begin{array}{l}\mathrm{L}+\mathrm{L}+\mathrm{L}+\mathrm{L}+\mathrm{L} \\
+\mathrm{WH}+\mathrm{RT}+\mathrm{L}\end{array}$ & $\begin{array}{l}\text { Clarified butter(Ghee) of Cow } \\
\text { milk;honey }\end{array}$ & $\begin{array}{l}\text { Alltheingredientsaregrindedtoforma } \\
\text { paste whichismixed well with Ghee. }\end{array}$ & $\begin{array}{l}\text { One teaspoonful } \\
\text { of mixture is } \\
\text { taken before } \\
\text { breakfast for } 4 \\
\text { to } 5 \text { days. }\end{array}$ \\
\hline
\end{tabular}




\begin{tabular}{|c|c|c|c|c|c|}
\hline Formulations & $\begin{array}{l}\text { Plant species used } \\
\text { singly / combinations }\end{array}$ & Parts used & Additives & Method of preparation & $\begin{array}{c}\text { Mode of } \\
\text { administration }\end{array}$ \\
\hline PF4 & $\begin{array}{c}\text { A bidentata }+ \\
\text { A Mexicana }+ \\
\text { B diffusa }+ \\
\text { B ceiba }+ \\
\text { B pinnatum }+ \\
\text { C viviparum }+ \\
\text { Corchioides }+B \text { lupulina }\end{array}$ & $\begin{array}{c}\mathrm{R}+\mathrm{R}+\mathrm{L}+\mathrm{L} \\
+\mathrm{L}+\mathrm{BU}+\mathrm{RH}+\mathrm{L}\end{array}$ & $\begin{array}{c}\text { Kalojira(Seeds ofNigellasativaL.; } \\
\text { Ranunculaceae); Rabing(FruitsofPiper } \\
\text { nigrumL.; Piperaceae); Michri(Sugar } \\
\text { candy) }\end{array}$ & Paste & $\begin{array}{l}\text { Apply on the } \\
\text { affected part }\end{array}$ \\
\hline PF5 & $\begin{array}{c}\text { C anthemoides } \\
+ \text { C reflexa }+ \\
\text { E scaber }+ \\
\text { F hispida }+ \\
\text { G arborea }+ \text { A spinosus }\end{array}$ & $\begin{array}{c}\mathrm{WH}+ \\
\mathrm{WH}+\mathrm{R}+\mathrm{L}+\mathrm{B}+\mathrm{A}\end{array}$ & $\begin{array}{l}\text { Blackpepper/Rabing(FruitsofPiper } \\
\text { nigrumL.; Piperaceae); Cummin/ } \\
\text { Jira(Seeds of CuminumcyminumL.; } \\
\text { Apiaceae); Milk }\end{array}$ & $\begin{array}{c}\text { A decoction is prepared in boiled } \\
\text { water }\end{array}$ & $\begin{array}{c}\text { Consumed with } \\
\text { ginger. }\end{array}$ \\
\hline PF6 & $\begin{array}{c}\text { M longifolia }+ \\
P \text { santalinus }+ \\
S \text { cordifolia }+ \\
\text { U indica }+ \\
\text { Canthemoides }+ \\
\text { A indica }+ \\
\text { Corchioides }+ \text { G bidens }+B \\
\text { purpurea }\end{array}$ & $\begin{array}{c}\mathrm{FR}+\mathrm{B}+\mathrm{L}+\mathrm{BU} \\
+\mathrm{WH}+\mathrm{L}+\mathrm{Rh}+ \\
\mathrm{WH}+\mathrm{L}\end{array}$ & Rice washed water & $\begin{array}{l}\text { All the materials are mixed together } \\
\text { to make a dough. Cakes/ pies are } \\
\text { prepared from the dough }\end{array}$ & $\begin{array}{l}\text { Cakes/pies are } \\
\text { consumed in } \\
\text { hot or warm } \\
\text { condition }\end{array}$ \\
\hline PF7 & $\begin{array}{c}\text { C viviparum }+ \\
\text { Corchioides }+ \\
\text { C rotundus }+ \\
\text { E hirta }+ \\
\text { A paniculata }+S \\
\text { zeylanica } P \text { crotalaroides }\end{array}$ & $\begin{array}{c}\mathrm{BU}+\mathrm{R}+ \\
\mathrm{WH}+\mathrm{A}+\mathrm{L}+\mathrm{RT}+\mathrm{RT}\end{array}$ & $\begin{array}{c}\text { Ada/Ginger } \\
\text { (RhizomeofZingiberofficinaleRoscoe; } \\
\text { Zingiberaceae): adequate } \\
\text { amount; Honey, equalto the } \\
\text { amountofGinger; Ricewashedwater }\end{array}$ & $\begin{array}{l}\text { All ingredients are grinded to a } \\
\text { paste with the help of a mortar and } \\
\text { pestle. Peas-like pills are made from } \\
\text { this paste }\end{array}$ & $\begin{array}{l}\text { Two pills are } \\
\text { taken each } \\
\text { day in empty } \\
\text { stomach for } 21 \\
\text { days }\end{array}$ \\
\hline PF8 & $\begin{array}{c}\text { H indicus }+ \\
C \text { viviparum }+ \\
\text { F bengaalensis }+G \\
\quad \text { superba } \\
+L \text { inermis }+ \\
\text { Corchioides }\end{array}$ & $\mathrm{A}+\mathrm{BU}+\mathrm{L}+\mathrm{L}+\mathrm{L}+\mathrm{RH}$ & $\begin{array}{c}\text { Bark ofmango/Aamtree } \\
\text { (MangiferaindicaL.; Anacardiaceae); } \\
\text { Chun }\end{array}$ & Paste & $\begin{array}{l}\text { Apply on } \\
\text { Carbuncle for } \\
7 \text { days }\end{array}$ \\
\hline PF9 & $\begin{array}{l}\text { M indica } \\
+P \text { betle } \\
+S \text { robusta } \\
+T \text { patula } \\
+T \text { indica }+ \\
\text { Canthemoides }\end{array}$ & $\mathrm{L}+\mathrm{L}+\mathrm{L}+\mathrm{L}+\mathrm{FR}+\mathrm{RH}$ & Cow urine & Mixed all to form paste & $\begin{array}{l}\text { Apply on the } \\
\text { affected part }\end{array}$ \\
\hline
\end{tabular}

$\mathrm{L}=$ Leaves; $\mathrm{BU}=\mathrm{Bulb} ; \mathrm{FR}=$ Flower; $\mathrm{RH}=$ Rhizome; $\mathrm{A}=$ Aerial part; $\mathrm{WH}=$ Whole plant; $\mathrm{B}=\mathrm{Bark} ; \mathrm{R}=$ Root; $\mathrm{IA}=$ Inflorescence axis; $\mathrm{LA}=$ Latex; $\mathrm{NR}=\mathrm{Not}$ required.

infection (0.6) (Table 3). High FL designates a plant's ethno-medicinal usage agreed upon by a number of informants.

\section{FL of the plants}

Plants were classified according to specific disease concern and FL value.

Boil $\div$

Achyranthes aspera L. (24\%), Allium sativum L. (28.57\%), Annona squamosa L. (33.33\%), Artocarpus heterophyllus Lam. (25\%), Azadirachta indica A. Juss. (34.04\%), Bryophyllum pinnatum (18.75\%), Cotula anthemoides L. (27.77\%), Crinum latifolium Linn. (46.66\%), Curculigo orchioides Gaertn. (12\%), Cuscuta reflexa Roxb. (48.27\%), Elephantopus scaber L. (23.07\%), Ficus hispida L.f. (23.52\%), Ficus racemosa L. (16.66\%), Hibiscus rosa-sinsnsis L. (28.57\%), Madhuca longifolia (Konig) J.F.Macbr. (16.66\%), Piper betle L. (51.61\%), Pterocarpus santalinus L.f. (15\%), Ricinus communis L., (24\%), Shorea robusta Gaertn., (23.07\%), Sida cordifolia L. (33.33\%), Tamarindus indica L., (40\%), Urginea indica (Roxb.) Kunth (29.16\%), Vitex negundo L. $(36.84 \%)$.

\section{Ulcer :}

Achyranthes aspera L. (28\%), Allium sativum L. (14.28\%), Andrographis paniculta (Burm.f.) Wal.ex. Nes. (59.25\%), Averrhoa carambola L. (33.33\%), Azadirachta indica A. Juss. (19.14\%), Cotula anthemoides L. (25.92\%), Curcuma longa L. (18.75\%), Cyperus rotundus L. (55.17\%), Elephantopus scaber L. (15.38), Hemidesmus indicus (L.) R.Br. (20.68\%), Madhuca longifolia (Konig) J.F.Macbr. (13.88\%), Urginea indica (Roxb.) Kunth (18.75\%), Aloe vera L. (17.64).

\section{Measles:}

Achyranthes bidentata Blume (26.66\%), Azadirachta indica A. Juss. (6.38\%), Euphorbia hirta L. (23.07\%).

\section{Cut}

Aloe veraL. (8.8\%), Argemone mexicana Linn (38.46\%), Boerhavia diffusa L. (45.83\%), Curculigo orchioides Gaertn. (18\%), Curcuma longa L. (15.62\%), Euphorbia hirta L. (19.23\%), Hemidesmus indicus (L.) R.Br. (6.66\%), Lawsonia inermis L. (15.38\%), Ricinus communis L. (28\%), Sida cordifolia L. (20\%), Syzygium cumini (L) Skeels (33.33), Tagetes patula L. 
Table 3: Data analyses for Informants' consensus factor for specific disease category.

\begin{tabular}{|c|c|c|c|c|c|}
\hline Category of ailments & Number of taxa & $\%$ of all taxa & Number of use reports & $\%$ all use reports & $\begin{array}{c}\text { Informants' consensus } \\
\text { factor (Fic) }\end{array}$ \\
\hline Acne & 6 & 3.57 & 32 & 2.56 & 0.84 \\
\hline Boil & 28 & 16.66 & 214 & 17.12 & 0.87 \\
\hline Burn & 10 & 5.95 & 53 & 4.24 & 0.83 \\
\hline Carbuncle & 58 & 34.52 & 597 & 47.76 & 0.91 \\
\hline Cut & 17 & 10.11 & 113 & 9.04 & 0.86 \\
\hline Chicken pox & 1 & 0.59 & 5 & 0.4 & 1 \\
\hline Eczema & 9 & 5.35 & 44 & 3.52 & 0.81 \\
\hline Leukoderma & 5 & 2.97 & 19 & 1.52 & 0.77 \\
\hline Measles & 4 & 2.38 & 13 & 1.04 & 0.75 \\
\hline Mouth ulcer & 1 & 0.59 & 1 & 0.08 & 0 \\
\hline Nail infection & 5 & 2.98 & 11 & 0.88 & 0.6 \\
\hline Pimple & 4 & 2.38 & 12 & 0.96 & 0.73 \\
\hline Ulcer & 14 & 8.33 & 104 & 8.32 & 0.87 \\
\hline Wound & 6 & 3.57 & 32 & 2.56 & 0.84 \\
\hline
\end{tabular}

(45.94\%), Tamarindus indica L. (28.88\%), Urginea indica (Roxb.) Kunth (12.5\%), Vitex negundo L. (26.31\%).

\section{Acne:-}

Aloe vera L. (11.76\%), Cotula anthemoides L. (11.11\%), Curcuma longa L. (21.87\%), Hibiscus rosa-sinsnsis L. (30.61\%).

\section{Nail infection $\div$}

Aloe vera L. (5.8\%), Datura metel L. (23.33), Datura stramonium L. (66.66\%), Ficus religiosa L. (50\%), Psidium guajava L. (8.33\%).

\section{Pimple :}

Annona squamosa L. (16.66\%), Cotula anthemoides L. (9.2\%), Ficus benghalensis L. (66.66).

\section{Chicken pox :}

Azadirachta indica A. Juss. (10.63\%)

\section{Mouth ulcer:}

Bombax ceiba L. (7.6\%)

\section{Burn $\div$}

Calotropis gigantea (Linn.) R. Br.ex. Ait. (47.82\%), Calotropis procera (Aiton) R. Br. (25\%), Curculigoorchioides Gaertn. (16\%), Elephantopus scaber L. (19.23\%), Euphorbia milii var. longifolia D. Moul. (40\%), Ficushispida L.f. (11.76\%), Ficus racemosa L. (37.5\%), Madhucalongifolia (Konig) J.F.Macbr. (27.77).

\section{Leukoderma}

Artocarpus heterophyllus Lam. (25\%), Curculigo orchioides Gaertn. (14\%), Gloriosa superba Linn. (7.1\%), Urginea indica (Roxb.) Kunth (10.41\%).

\section{Wound $\div$}

Gmelina arborea Roxb. Ex Sm. (13.33\%), Mangifera indica L. (20\%), Pterocarpus santalinus L.f. (15\%), Shorearobusta Gaertn. (19.23\%), Syzygium cumini (L) Skeels (35.89\%).

\section{Eczema :}

Hemidesmus indicus (L.) R.Br. (37.93\%), Mangifera indica L. (24\%), Pterocarpus santalinus L.f. (15\%), Shorea robusta Gaertn. (19.23\%), Syzygium cumini (L) Skeels (35.89\%), Tagetes patula L. (16.21\%), Sida cordata (Burm. f.) Borss. Waalk. (30\%).

\section{Carbancle:}

Achyranthes aspera L. (48\%), Achyranthes bidentata Blume (73.33\%), Allium sativum L. (66.66\%), Aloe vera L. (55.88\%), Andrographis paniculta (Burm.f.) Wal.ex.Nes. (40.74\%), Annona squamosa L. (50\%), Argemone mexicana Linn (61.53\%), Artocarpus heterophyllus Lam. (50\%), Averrhoa carambola L. (66.66\%), Azadirachta indica A. Juss. (29.78\%), Boerhavia diffusa L. (54.16\%), Bombax ceiba L. (92.30\%), Bryophyllum pinnatum (Lam) Oken (81.25\%), Calotropis gigantea (Linn.) R.Br.ex.Ait. (52.17\%), Calotropis procera (Aiton) R. Br. (75\%), Cotula anthemoides L. (25.92\%), Crinum viviparum (Lam) R. Ansari \& V.J. Nair, Curculigo orchioides Gaertn. (40\%), Curcumalonga L. (43.75\%), Cuscuta reflexa Roxb. (51.72\%), Cyperus rotundus L. (44.82\%), Datura metel L. (66.66\%), Datura stramonium L. (33.33), Elephantopus scaber L. (42.30), Euphorbia hirta L. (61.53\%), Euphorbia milii var. longifolia D. Moul. (60\%), Ficus benghalensis L. (33.33\%), Ficus hispida L.f. (64.70\%), Ficus racemose L. (45.83\%), Ficus religiosa L. (50\%), Gloriosa superba Linn. (92.85\%), Gmelina arborea Roxb. ex Sm. (80\%), Hemidesmus indicus (L.) R.Br. (41.37\%), Hibiscusrosa-sinsnsis L. (40.81\%), Lawsoniai nermis L. (84.61\%), Madhucalongifolia (Konig) J.F.Macbr. (41.66\%), Mangifera indica L. (56\%), Piper betle L. (48.38\%), Psidium guajava L. (58.33\%), Pterocarpus santalinus L.f. (52.5\%), Ricinus communis L. (48\%), Shorea robusta Gaertn. (42.30\%), Sida cordata (Burm. f.) Borss. Waalk. (70\%), Sida cordifolia L. (46.66\%), Syzygium cumini (L)Skeels (30.76\%), Tagetes patula L. (37.83\%), Tamarindus indica L. (31.11\%), Urginea indica (Roxb.) Kunth (29.16\%), Vitex negundo L. (36.84\%).

\section{DISCUSSION AND CONCLUSION}

We report a total of 59 plant species belonging to 35 different families which are used directly or with some additives (Table 2) for the treatment of carbuncle. Herbal formulations are usually made with dry dehydrated form rather than fresh form of plant parts. Dried plant parts are stored at homes throughout the year so that these can be used for treatment during off-season, especially in winter when leafy species become scarce. Usually, healers follow their own traditional knowledge for drug formulation and do not want to disclose it to the patients. In most of the cases they prefer leafy parts of different herbs. UV represents the relative importance of plants in that locality for drug formulation (Table 1). High Fic designates the use of a particular species reported by a large fraction of the interviewed informants for a particular disease and low Fic denotes the disparity amongst the informants in using a specific species against a specific disease. In many cases, medication was preferred in a composite mixtures from different plants/plant parts with the understanding that synergistic effect of phytochemicals of 
different plants species may improve the rate of healing. ${ }^{20}$ This classical knowledge, inherited by these local tribal healers from their ancestors is rapidly vanishing due to degradation of forest covers, uprooting of tribal population due to fast urbanization and industrialization and above all indifferent attitude of younger generation, although still maintaining the skeletal structure of primary healthcare system of Purulia district. Therefore, the present documentation is a preliminary attempt to pave the path for developing digitized database in future. A proper planning and management is the need of the age for their sustainable exploitation and conservation.

\section{ACKNOWLEDGEMENT}

The authors gratefully acknowledge the U.G.C. for financial support from U.G.C. minor research project no. F.PSW-204/15-16(ERO). We are indebted to the tribal people of Purulia who helped us a lot to give the manuscript a full shape. Their immense help inspire us to project their traditional knowledge globally through publication. GM, first author is grateful to the Principal, A.M. College, Purulia for giving permission for field work.

\section{CONFLICTS OF INTEREST}

None.

\section{ABBREVIATIONS}

FL: Fidelity Level; Fic: Informants' Consensus Factor; UV: Use Value; MF: Monoherbal Formulation; PF: Polyherbal Formulation.

\section{REFERENCES}

1. Abbasi AM, Khan MA, Ahmad M, Zafar M, Jahan S, Sultana S. Ethno pharmacological application of medicinal plants to cure skin diseases and in folk cosmetics among the tribal communities of North-West Frontier Province, Pakistan. J Ethnopharmacol. 2010;128(2):322-35.

2. Quave $C L$, Pieroni $A$, Bennett $B C$. Dermatological remedies in the traditional pharmacopoeia of Vulture-Alto Bradano, inland southern Italy. J Ethnobiol Ethnomed. 2008;4(1):5

3. Adetutu A, Witson AM, Corcoran O. Ethnopharmacological survey and in vitro evaluation of wound healing plants used in South-western Nigeria. J Ethnopharmacol. 2011;137(1):50-6.

4. Saikia AP, Ryakala VK, Sharma P, Goswami P, Bora U. Ethnobotany of medicinal plants used by Assamese people for various skin ailments and cosmetics. J Ethnopharmacol. 2006;106(2):149-57.

5. World Heritage Encyclopedia,http://www.worldebooklibrary.org/articles/ Carbuncle;World e-bookLibrary. ID-WHEBN0000712333;CarbunclePubmed,2011.

6. Bowersox J. Experimental staph vaccine broadly protective in animal studies NIH.1999. Archived from the original on 5 May 2007. Retrieved 28 July 2007.

7. Jevons MP. Celbenin-resistant staphylococci. BMJ.1961;1(5219):124-5.

8. Chambers HF. The changing epidemiology of Staphylococcus aureus? Emerg Infect Dis. 2001;7(2):178-82.

9. Hiramatsu K, Hanaki H, Ino T, Yabuta K, OguriT, Tenover FC. Methicillin-resistant Staphylococcus aureus clinical strain with reduced vancomycin susceptibility (PDF). J Antimicrob Chemother. 1997:40(1):135-6.

10. Okemo PO, Bais HP, Vivanco JM. In vitro activities of Maesa lanceolata extracts against fungal plant pathogens. Fitoterapia. 2003;74(3):312-6.

11. Paksoy MY, Selvi S, Savran A. Ethnopharmacological survey of medicinal plants in Ulukisla. J Herbal medicine. 2016;1-7.

12. Modak BK, Gorai P, Dhan R, Mukherjee A, Dey A. Tradition in treating taboo: folkloric medicinal wisdom of the aboriginals of Purulia district, West Bengal India against sexual gynaecological and related disorders. J Ethnopharmacol. 2015; 169:370-86.

13. Bentham G, Hooker JD. Genera Planterum. London: Lovell Reeve and Co. 1862-83;1-3.

14. Prain D. Bengal plants. Dehra Dun: Bishen Singh Mahendra Pal Singh. 1903;1-2. 15. Pal DC, Jain SK. Tribal medicine. Kolkata: Naya Prakash. 1998

16. Paria ND, Chattopadhyay SP. Flora of Hazaribagh district, Bihar. Bot Survey of India. 2005;2:1299.

17. Trotter RT, Logan $\mathrm{MH}$. Informant census: A new approach for identifying potentially effective medicinal plants. In: Etkin, L.N. (Ed.), Plants in indigenous medicine and diet. Redgrave, Bedford Hill, New York. 1986;91-112.

18. Friedmen J, Yaniv Z, Dafni A, Palewitch D. A preliminary classification of the healing potential of medicinal plants, based on a rational analysis of an ethno pharmacological field survey among Bedouins in the Negev desert, Israel. J Ethnopharmacol. 1986;16(2-3):275-87.

19. Phillips O, Gentry AH, Reynel C, Wilkin P, Galvez-Durand BC. Quanti-tative ethno botany and amazonian conservation. Conserv Biol. 1994;8(1):225-48.

20. Gertsch J. Botanical drugs, synergy and network pharmacology: Forth and back to intelligent mixtures. Planta Med. 2011;77(11):1086-98.

\section{SUMMARY}

\section{GRAPHICAL ABSTRACT}

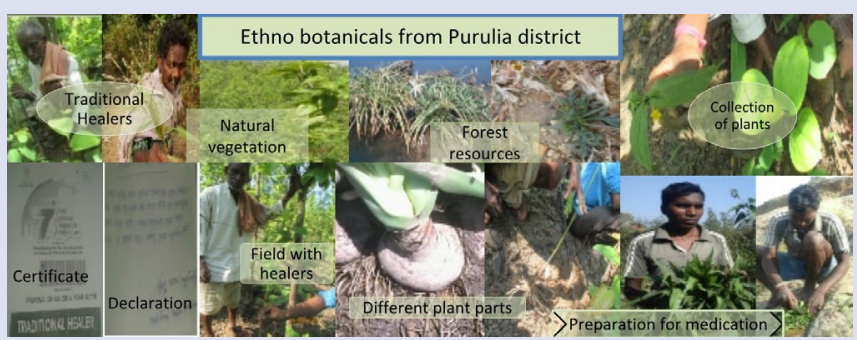

Fifty-nine herbal plants belongs to 35 families were used in various combinations during treatment. Most of the species belongs to Moraceae and Asteraceae families in comparison to other families. Herbaceous plants are most commonly used and leaves are the most common part for preparing formulations.

\section{ABOUT AUTHORS}

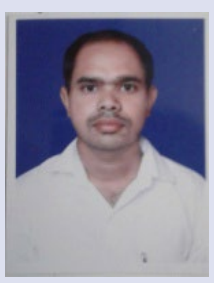

Ghanashyam Mahato: Assistant Professor, Department of Botany, A.M. College, Jhalda, Purulia, West Bengal-723202, India. 
Bangamoti Hansda: Research Scholar, Department of Botany and Forestry, Vidyasagar University, Midnapore, West Bengal-721102, India.

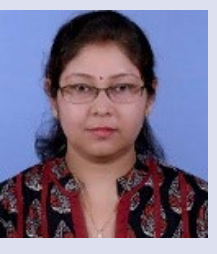

Nilanjana Banerjee: Assistant Professor, Department of Botany, Vidyasagar University, Paschim Medinipur, West Bengal-721102, India.

Cite this article: Mahato G, Hansda B, Banerjee N. Ethnobotanicals used for the Treatment of Skin Diseases with Special Emphasis on Carbuncle Disease from Purulia District of West Bengal in India. Pharmacog J. 2019;11(4):745-53. 